\title{
LXIII. The differential equation of the most general substitution of one variable
}

\section{Captain P.A. MacMahon R.A.}

To cite this article: Captain P.A. MacMahon R.A. (1887) LXIII. The differential equation of the most general substitution of one variable , Philosophical Magazine Series 5, 23:145, 542-543, DOI: $10.1080 / 14786448708628049$

To link to this article: http://dx.doi.org/10.1080/14786448708628049

Published online: 29 Apr 2009.

Submit your article to this journal $₫$

Џ Article views: 2

Q View related articles $\square$ 
LXIII. The Differential Equation of the most general substitution of one Variable. By Captain P. A. MacMAHon, R.A.*

T the Philosophical Magazine for February 1886, Dr. T. 1 Muir considers the differential equations of the general conic and cubic curves by a perfectly general method.

The general linear substitution

$$
y=\frac{(a, b)(x, 1)}{\left(a^{\prime}, b^{\prime}\right)(x, 1)}
$$

leads, as is well known, to the differential equation

$$
2 \frac{d y}{d x} \cdot \frac{d^{3} y}{d x^{3}}-3\left(\frac{d^{2} y}{d x^{2}}\right)^{2}=0,
$$

wherein the expression on the left has been called the Schwarzian derivative : this is a reciprocant; but it is also an invariant, as may be seen by writing

$$
\frac{d y}{d x}=1 ! t, \quad \frac{d^{2} y}{d x^{2}}=2 ! a, \quad \frac{d^{3} y}{d x^{3}}=3 ! b,
$$

when it assumes the form

$$
12\left(t b-a^{2}\right) \text {. }
$$

In the case of the general substitution of order $n$, the resulting expression is no longer a reciprocant, but it is an invariant (catalecticant) of a certain binary quantic $\dagger$.

For, writing

we have

$$
y=\frac{(a, b, c, \ldots)(x, 1)^{n}}{\left(a^{\prime}, b^{\prime}, c^{\prime}, \ldots\right)(x, 1)^{n}}=\frac{\mathrm{U}_{n}}{\mathrm{~V}_{n}}
$$

$$
y \mathrm{~V}_{n}=\mathrm{U}_{n}
$$

Differentiating this equation $n+1, n+2, n+3, \ldots 2 n+1$ times successively by Leibnitz's theorem, and putting

$$
\frac{d^{p} y}{d x^{p}}=y_{p}, \quad \frac{d^{p} \mathrm{~V}_{n}}{d x}=\mathrm{V}_{n}^{(p)}
$$

there results the set of equations :-

* Communicated by the Author.

$\dagger$ The formation of the differential equation was recently set as a question in an examination for Fellowship at Trinity College, Dublin; but I am not aware that its connexion with the theory of Invariants has been before noticed. 
The Differential Equation of the general Substitution. 543

$$
\begin{aligned}
& y_{n+1} \mathrm{~V}_{n}+(n+1) y_{n} \mathrm{~V}_{n}^{(1)}+\frac{(n+1) !}{2 !(n-1) !} y_{n-1} \mathrm{~V}_{n}^{(2)}+\ldots+\frac{(n+1) !}{n ! 1 !} y_{1} \mathrm{~V}_{n}^{(n)}=0 \\
& y_{n+2} \mathrm{~V}_{n}+(n+2) y_{n+1} \mathrm{~V}_{n}^{(1)}+\frac{(n+2) !}{2 ! n !} y_{n} \quad \mathrm{~V}_{n}^{(2)}+\ldots+\frac{(n+2) !}{n ! 2 !} y_{2} \mathrm{~V}_{n}^{(n)}=0 \\
& y_{n+3} \mathrm{~V}_{n}+(n+3) y_{n+2} \mathrm{~V}_{n}^{(1)}+\frac{(n+3) !}{2 !(n+1) !} y_{n+1} \mathrm{~V}_{n}^{(2)}+\ldots+\frac{(n+3) !}{n ! 3 !} y_{3} \mathrm{~V}_{n}^{(n)}=0 \\
& y_{2 n+1} \mathrm{~V}_{n}+(2 n+1) y_{2 n} \mathrm{~V}_{n}^{(1)}+\frac{(2 n+1) !}{2 !(2 n-1) !} y_{2 n-1} \mathrm{~V}_{n}^{(2)}+\ldots+\frac{(2 n+1) !}{n !(n+1) !} y_{n+1} \mathrm{~V}_{n}^{(n)}=0 \\
& \text { or writing } \\
& y_{1}=1 ! t, \quad y_{2}=2 ! a_{0}, \quad y_{3}=3 ! a_{1}, \ldots y_{p}=p ! a_{p-2}, \\
& t \frac{1}{n !} \nabla_{n}^{(n)}+a_{0} \frac{1}{(n-1) !} \mathrm{V}_{n}^{(n-1)}+a_{1} \frac{1}{(n-2) !} \nabla_{n}^{(n-2)}+\ldots+a_{n-1} \mathrm{~V}_{n}=0 \\
& a_{0} \frac{1}{n !} \mathrm{V}_{n}^{(n)}+a_{1} \frac{1}{(n-1) !} \mathrm{V}_{n}^{(n-1)}+a_{2} \frac{1}{(n-2) !} \mathrm{V}_{n}^{(n-2)}+\ldots+a_{n} \quad \mathrm{~V}_{n}=0 \\
& a_{1} \frac{1}{n !} \mathrm{V}_{n}^{(n)}+a_{2} \frac{1}{(n-1) !} \mathrm{V}_{n}^{(n-1)}+a_{3} \frac{1}{(n-2) !} \nabla_{n}^{(n-2)}+\ldots+a_{n+1} \mathrm{~V}_{n}=0 \\
& a_{n-1} \frac{1}{n !} \mathrm{V}_{n}^{(n)}+a_{n} \frac{1}{(n-1) !} \mathrm{V}_{n}^{(n-1)}+a_{n+1} \frac{1}{(n-2) !} \mathrm{V}_{n}^{(n-2)}+\ldots+a_{2 n-1} \nabla_{n}=0
\end{aligned}
$$

Eliminating the $n+1$ quantities,

$$
\frac{1}{n !} \mathrm{V}_{n}^{(n)}, \frac{1}{(n-1) !} \mathrm{V}_{n}^{(n-1)}, \ldots \mathrm{V}_{n}
$$

between these $n+1$ equations, we find that the desired differential equation is

$$
\left|\begin{array}{cccccc}
t & a_{0} & a_{1} & \ldots & a_{n-2} & a_{n-1} \\
a_{0} & a_{1} & a_{2} & \ldots & a_{n-1} & a_{n} \\
a_{1} & a_{2} & a_{3} & \ldots & a_{n} & a_{n+1} \\
\vdots & \vdots & \vdots & \vdots & \vdots & \vdots \\
a_{n-1} & a_{n} & a_{n+1} & \ldots & a_{2 n-2} & a_{2 n-1}
\end{array}\right|=0 .
$$

This determinant is the catalecticant of the binary quantic

$$
\left(t, a_{0}, a_{1}, \ldots a_{2 n-1}\right)(\mathrm{X}, \mathrm{Y})^{2 n}
$$

and by counting the constants, we see that the general substitution is the complete primitive of the differential equation.

Royal Military Academy, Woolwich, May 21st, 1887. 